\title{
25 Research Soure \\ Validity and Reliability of the Japanese Version of the Cognitive Scale for Functional Bowel Disorders
}

\section{Nagisa Sugaya ( $\nabla$ nagisa618@gmail.com )}

Yokohama City University: Yokohama Shiritsu Daigaku https://orcid.org/0000-0002-4803-3907

\section{Yoshitoshi Tomita}

National Center of Neurology and Psychiatry: Kokuritsu Seishin Shinkei Center

\section{Misako Funaba}

National Center of Neurology and Psychiatry: Kokuritsu Seishin Shinkei Center

Hiroshi lida

Yokohama City University: Yokohama Shiritsu Daigaku

Kentaro Shirotsuki

Musashino University: Musashino Daigaku

Fumiyuki Chin Gardner

The Pennsylvania State University

\section{Tetsuya Ando}

International University of Health and Welfare: Kokusai Iryo Fukushi Daigaku

\section{Masahiko Inamori}

Yokohama City University: Yokohama Shiritsu Daigaku

\section{Research Article}

Keywords: functional gastrointestinal disorder, irritable bowel syndrome, Cognitive Scale for Functional Bowel Disorders

Posted Date: December 13th, 2021

DOl: https://doi.org/10.21203/rs.3.rs-1112940/v1

License: (c) (i) This work is licensed under a Creative Commons Attribution 4.0 International License.

Read Full License 


\section{Abstract}

\section{Background}

The Cognitive Scale for Functional Bowel Disorders (CS-FBD) is a useful measure to assess maladaptive cognition, and focuses on how functional bowel disorders relate to negative thoughts, perfectionism, and social desirability. This study aimed to confirm the reliability and validity of the Japanese version of the CS-FBD (CS-FBD-J).

\section{Methods}

Participants comprised 192 students $(20.2 \pm 3.0$ years $)$ and 22 outpatients diagnosed with irritable bowel syndrome (IBS) (38.0 \pm 13.0 years). There were 76 students who met the diagnostic criteria for IBS, and two students who received treatment for IBS. Participants completed questionnaires containing the CSFBD-J, IBS Severity Index (IBS-SI), Visceral Sensitivity Index (VSI), 24-item Dysfunctional Attitudes Scale (DAS-24), and Hospital Anxiety and Depression Scale (HADS).

\section{Results}

Our factor analysis revealed that the CS-FBD-J had a unidimensional factor structure, and that the factor loadings for 2 out of the 25 items were less than 0.4. After excluding the two items from the analysis, a single factor of the 23 -item version accounted for $45.85 \%$ of the total variance. The CS-FBD-J scores had significant moderate correlations with the IBS-SI $(r=0.492 \sim 0.574)$ and VSI $(r=0.531 \sim 0.557)$ scores in the IBS group and the control group. Correlation between the DAS-24 and the CS-FBD-J was not significant $(r=0.179 \sim 0.191)$. Although the CS-FBD-J in the IBS group was significantly correlated with HADS-anxiety $(r=0.450)$ and depression scores $(r=0.357)$, their intercorrelations in the control group were not significant ( $r=0.150 \sim 0.167)$. In the score comparison of the CS-FBD-J between the IBS patient group, non-patient IBS group (students with IBS except two who received treatment), and control group, there were significant group effects in the CS-FBD-J (IBS patient > non-patient IBS > control). The internal consistencies of the CS-FBD-J were high $(a=0.95)$. The item-total correlation analysis for the CS-FBD-J showed that the correlations between each item and the total score were significant.

\section{Conclusion}

This study confirmed the reliability and validity of the 23-item version of the CS-FBS with the deletion of two items with low factor loadings.

\section{Background}

Irritable bowel syndrome (IBS) is a functional gastrointestinal disorder characterized by persistent chronic abdominal pain and disturbance of bowel movements. It is a typical psychosomatic disorder of the digestive system that is exacerbated by psychological stress. Many previous studies have reported the effect of cognitive behavioral therapy $\mathrm{CBT}$ ) or cognitive therapy on abdominal symptoms and emotional 
distress in patients with IBS [1,2]. Our previous study [3] suggested that the cognitive-emotion process is related to the aggravation of anxiety in individuals with IBS; severe anxiety sensitivity in individuals with IBS related to their symptom-related cognition and the altered cognition associated with increased anxiety can lead to the development of a disabling condition including avoidant behavior.

The Cognitive Scale for Functional Bowel Disorders (CS-FBD [4]) is a useful measure to assess maladaptive cognitions, focusing on functional bowel disorders related to negative thoughts, perfectionism, and social desirability. The CS-FBD is a valid and reliable scale that can be used as an outcome measure in evaluating the efficacy of different forms of psychotherapeutic intervention for functional bowel disorders, including IBS, and can also serve as a helpful assessment tool for health professionals working with patients diagnosed with IBS [4]. CS-FBD has been used in many previous studies, including clinical trials of CBT [5-10]. The Visceral Sensitivity Index (VSI [11]) has been developed to assess gastrointestinal specific anxiety, cognitive, affective, and behavioral responses to fear of GI sensations, symptoms, and the context in which they occur, and it has been translated into Japanese [12]. The CS-FBD focuses on maladaptive cognition that is not specific to anxiety, and its content is differentiated from the VSI. CS-FBD items were generated from the diaries of automatic thought records in patients with functional gastrointestinal disorders, and included a variety of themes: bowel performance anxiety, out of control, perfectionism, anger/frustration, self-efficacy, social approval, embarrassment/shame, heightened sensitivity to social rules and norms, and self-nurturance. The CSFBD was utilized along with the VSI in previous studies (e.g., [9]). However, a Japanese version of the CSFBD has not yet been developed.

Therefore, the purpose of this study is to confirm the reliability and validity of the Japanese version of the CS-FBD (CS-FBD-J).

\section{Methods}

\section{Participants}

Participants were recruited between September 2013 and March 2021. Participants of the present study were outpatients who were diagnosed with IBS in the gastroenterology and psychosomatic medicine departments of hospitals in Tokyo and Kanagawa and college students, including IBS patients or nonpatient IBS who met diagnostic criteria (Rome III criteria) and those who did not meet the diagnostic criteria. The present study was approved by the ethical committee of Yokohama City University (B130905050) and the National Center of Neurology and Psychiatry (A2018-046). Written informed consent was obtained from all participants.

\section{Measures}

\section{Cognitive Scale for Functional Bowel Disorders translated in Japanese [4]}


The Cognitive Scale for Functional Bowel Disorders (CS-FBD) includes 25 items that measure maladaptive cognition related to abdominal symptoms, with responses ranging from 1 (strongly disagree) to 7 (strongly agree). The total score for this scale ranges from 25 to 175 . One of the authors (N.S.), who is a psychologist knowledgeable about functional gastrointestinal disorders and psychosomatic medicine, translated the original version into Japanese. Another author (F.G.) who is bilingual in English and Japanese, back-translated the Japanese items of the CS-FBD into English. We compared the results of the back translation to the original version and confirmed agreement among the researchers about the nuances.

\section{Japanese version of the Rome III modular questionnaire (only items related to IBS) [13,14]}

The Rome III Diagnostic Criteria for Functional Gastrointestinal Disorders [15] have been widely used to define IBS. Rome IV has not been published at the start of this investigation. Using the Rome III modular questionnaire, the presence of IBS was determined if the participants had abdominal pain or discomfort for at least 3 weeks (at least once per week) in the previous 3 months and two of the three following symptoms: (1) pain or discomfort that improves or stops after a bowel movement, (2) a change in the number of bowel movements when the pain or discomfort starts, and (3) either softer or harder stools than usual when the pain or discomfort starts.

\section{IBS Severity Index [16,17]}

The IBS Severity Index (IBS-SI) was used to assess the severity of gastrointestinal symptoms. This scale consists of 5 items: abdominal pain ( 2 items), abdominal distension, bowel movements, and quality of life, and the total score ranges from 0 to 500 . The IBS-SI classifies the severity of IBS as mild (75-174), moderate (175-299), or severe (300-500) based on clinical observations of patients with IBS.

\section{Japanese version of Visceral Sensitivity Index [11,12]}

The Visceral Sensitivity Index (VSI) is a self-reported measure of gastrointestinal symptom-specific anxiety in patients with IBS. The VSI includes 15 items with responses ranging from 1 (strongly agree) to 6 (strongly disagree), and scores ranging from 0 to 5.

\section{Japanese version of the 24-item Dysfunctional Attitudes Scale (Tajima et al., 2007 [18])}

The 24-item Dysfunctional Attitudes Scale (DAS-24) is a self-report scale for depression schemata with responses ranging from 1 (strongly disagree) to 7 (strongly agree). The DAS-24 contains three factors: "achievement," "self-control," and "dependency." The achievement subscale consists of eight items, for example, "If I fail partly, it is as bad as being a complete failure." The dependency subscale consists of eight items, for example, "I am nothing if a person I love doesn't love me." The self-control subscale consists of eight items, for example, "A person should do well at everything he undertakes."

\section{Hospital Anxiety and Depression Scale [19,20]}


The Hospital Anxiety and Depression Scale (HADS) is a self-report questionnaire for anxiety and depression. The HADS includes of 14 questions on a 4-point scale, consisting of an anxiety subscale with 7 items and a depression subscale with 7 items. This psychometric instrument was chosen because all its items refer solely to an emotional state and do not consider somatic symptoms.

\section{Procedure}

\section{Patients}

Patients with IBS were given written and oral explanations about the purpose of this study, the protection of their personal information, and that participation was voluntary. Patients with IBS who agreed to participate in this study provided informed consent. Participants then completed questionnaires containing the physical and psychological scales mentioned above.

\section{College students}

The researchers recruited college students after class hours or via the university's online bulletin board. Participants recruited by the latter method were limited to those who met the diagnostic criteria for IBS. They were given written and oral explanations about the purpose of this study, the protection of their personal information, that participation was voluntary, and that they would not be punished for not cooperating. After the above explanations were given, only those students who agreed to participate were asked to complete a series of questionnaires.

\section{Statistical analysis}

The factor structure of the CS-FBD-J was confirmed by exploratory factor analysis. Construct validity was confirmed using Pearson's correlation coefficient analysis between the CS-FBD-J and scores on the IBS-SI, VSI, DAS, and HADS. Criterion-related validity was confirmed using one-way analysis of variance to compare the CS-FBD-J score between IBS patient group, non-patient IBS group and control group, and a post-hoc test with Scheffe's method was employed to test the differences between groups. The internal consistency reliability of the CS-FBD-J was determined using Cronbach's a. The correlations between each item and the total score of the CS-FBD-J were used to conduct item-total correlation analysis. The significance level was set at less than $5 \%$. Data analyses were performed using SPSS software (version 25.0; IBM Corp., NY, USA).

\section{Results}

\section{Descriptive results}

Table 1 presents the descriptive results for the participants. In our study, 203 college students were enrolled, and 192 students ( $20.2 \pm 3.0$ yrs.) provided valid data. There were 76 students who met the diagnostic criteria for IBS, and 2 students received treatment for IBS. Twenty-two outpatients who were diagnosed with IBS in the gastroenterology and psychosomatic medicine departments of hospitals in 
Tokyo and Kanagawa (38.0 \pm 13.0 yrs.) provided valid data. Eleven participants who received treatment for IBS received psychiatric medication. The IBS group (IBS patients and non-IBS patients) included 28 participants with diarrhea-predominant IBS (IBS-D), 9 participants with constipation-predominant IBS (IBS-C), 55 participants with mixed type IBS (IBS-M), and 6 participants with unclassifiable IBS (IBS-U). Table 2 shows the differences in each variable between the IBS patient group, non-patient IBS group, and control group. There were significant group effects in the IBS-SI and VSI, and all comparisons between all groups in these variables except that between the IBS patient group and the non-IBS group in IBS-SI showed significant differences under multiple comparison analysis.

Table 1

Descriptive results of the study participants

\begin{tabular}{|llllll|}
\hline \multicolumn{5}{|c|}{ N\%) } \\
\hline & Total & Male & \multicolumn{3}{c|}{ Female } \\
\hline IBS patient & 24 & 17 & $(70.8)$ & 7 & $(29.2)$ \\
\hline Non-patient IBS & 74 & 21 & $(28.4)$ & 53 & $(71.6)$ \\
\hline Control & 116 & 32 & $(27.6)$ & 84 & $(72.4)$ \\
\hline IBS-D & 28 & 13 & $(46.4)$ & 15 & $(53.6)$ \\
\hline IBS-C & 9 & $(11.1)$ & 8 & $(88.9)$ & \\
\hline IBS-M & 55 & 22 & $(40.0)$ & 33 & $(60.0)$ \\
\hline IBS-U & 6 & 2 & $(33.3)$ & 4 & $(66.7)$ \\
\hline IBS: Irritable bowel syndrome & & & \\
\hline IBS-D: Diarrhea-predominant IBS & & & \\
\hline IBS-C: Constipation-predominant IBS & \\
\hline IBS-M: Mixed-type IBS & & \\
\hline IBS-U: Unclassifiable IBS
\end{tabular}


Table 2

Comparison of each variable between IBS patient, non-patient IBS, and control groups

\begin{tabular}{|c|c|c|c|c|c|c|c|c|c|}
\hline \multirow[b]{3}{*}{$\begin{array}{l}\text { CS-FBD (25 } \\
\text { items) }\end{array}$} & \multicolumn{9}{|c|}{ Mean (SD) } \\
\hline & \multicolumn{2}{|c|}{ IBS patient } & \multicolumn{2}{|c|}{$\begin{array}{l}\text { Non-patient } \\
\text { IBS }\end{array}$} & \multicolumn{2}{|c|}{ Control } & \multirow{2}{*}{$\begin{array}{l}F \\
44.53\end{array}$} & \multirow{2}{*}{$\begin{array}{l}p \\
< \\
0.001\end{array}$} & \multirow{2}{*}{$\begin{array}{l}\eta^{2} \\
0.297\end{array}$} \\
\hline & 103.5 & $(30.2)$ & 78.6 & $(25.1)$ & 57.1 & $(21.9)$ & & & \\
\hline $\begin{array}{l}\text { CS-FBD (23 } \\
\text { items) }\end{array}$ & 93.7 & $(29.2)$ & 69.8 & $(24.6)$ & 49.4 & $(20.7)$ & 43.79 & $<.001$ & 0.293 \\
\hline IBS-SI & 193.3 & (109.7) & 193.9 & $(80.7)$ & 116.1 & $(86.6)$ & 20.93 & $<.001$ & 0.166 \\
\hline VSI & 52.4 & $(14.7)$ & 23.2 & (19.8) & 13.6 & (17.0) & 46.10 & $<001$ & 0.331 \\
\hline DAS & 92.1 & $(24.4)$ & 93.1 & $(24.5)$ & 87.3 & $(20.7)$ & 1.63 & 0.198 & 0.015 \\
\hline HADS (Anxiety) & 7.4 & $(4.8)$ & 7.3 & (3.6) & 6.4 & (3.8) & 1.72 & 0.182 & 0.016 \\
\hline $\begin{array}{l}\text { HADS } \\
\text { (Depression) }\end{array}$ & 5.3 & $(4.8)$ & 4.8 & (3.0) & 4.9 & (3.8) & 0.17 & 0.843 & 0.002 \\
\hline HADS (Total) & 12.7 & (9.1) & 12.1 & $(6.0)$ & 11.3 & (7.0) & 0.60 & 0.552 & 0.006 \\
\hline
\end{tabular}

\section{Factor analysis}

Exploratory factor analysis (using maximum likelihood estimation and promax rotation) without assigning the number of factors revealed the presence of five components with eigenvalues exceeding 1 . However, inspection of the scree plot revealed a break after the first component. Therefore, we decided to retain a unidimensional factor structure for further investigation (Table 3). Factor analysis showed that the factor loadings for two items (items 6 and 18) were less than 0.4. After excluding these items from the analysis, the factor loadings for all items were above 0.4 , and a single factor of the 23 -item version accounted for $45.85 \%$ of the total variance. 
Table 3

Results of the item-total analysis and factor analysis

25-item version

Items

1. Not getting to bathroom in time

2. I'm always sick with bowel problems

3. Symptoms are too much to handle

4. Can't function normally when sick with bowel problems

5. Bowel symptoms are agony

0.763

6. Do my absolute best at everything

7. Frustrated by bowel symptoms

8. Pain will never go away

9. I feel very down about my bowel symptoms

10. Passing gas in public

11. Worry about not finding a bathroom when I need one

12. Bowel problems interfere with feeling good about myself

13. Worry about my bowel symptoms on a trip

14. Can't concentrate due to pain

15. Embarrassing to keep going to bathroom

16. Concern about lasting through events

17. Being late upsets me

0.471

0.774

0.323

0.722

0.700

0.712

0.761

0.789

0.767

0.611

0.802

0.567

0.389

0.720

19. Not take advantage of opportunities due to bowel problems

20. Symptoms make me feel out of control

21. Bowel symptoms in restaurant

0.639

0.686

0.724

0.693

0.726

0.769

0.763

0.769

0.732

0.262

0.754

0.734

0.755

0.727

0.759

0.730

0.414

0.473

0.712

0.776

0.763

0.763

0.800

0.767

0.766

0.780

0.576

0.581

0.607

0.817

0.782

0.781

0.541

0.472

0.480

0.727

0.786

0.785

0.724

0.635

0.653

0.636
Factor loadings

0.491

0.705

0.696

0.695 


\begin{tabular}{|lllll|}
\hline & \multicolumn{2}{l}{ 25-item version } & \multicolumn{2}{l|}{ 23-item version } \\
\hline $\begin{array}{l}\text { 22. With frequent bathroom visits, others } \\
\text { think something is wrong }\end{array}$ & 0.668 & 0.627 & 0.663 & 0.622 \\
\hline $\begin{array}{l}\text { 23. Worry about losing control of bowels } \\
\text { in public }\end{array}$ & 0.810 & 0.770 & 0.812 & 0.768 \\
\hline 24. Feeling guilt if I nurture myself & 0.431 & 0.452 & 0.433 & 0.450 \\
\hline $\begin{array}{l}\text { 25. Must get home when 1 have } \\
\text { symptoms }\end{array}$ & 0.553 & 0.541 & 0.563 & 0.542 \\
\hline & Eigenvalue & 10.72 & Eigenvalue & 10.54 \\
\hline & $\begin{array}{l}\text { Variance } \\
(\%)\end{array}$ & $42.87 \%$ & $\begin{array}{l}\text { Variance } \\
(\%)\end{array}$ & $45.85 \%$ \\
\hline
\end{tabular}

\section{Construct validity}

The results of the correlation analysis between the CS-FBD-J and other variables in the IBS and control groups confirmed the construct validity (Table 4). The 25 -item and 23 -item versions of CS-FBD-J scores had significant moderate correlations with the IBS-SI and VSI scores in all participants, the IBS group, and the control group. The DAS-24 score was significantly correlated with the 25 -item version of CS-FBD-J in both the IBS and control groups, but the correlations were low. Moreover, correlations between the 23-item version of CS-FBD-J and DAS-24 did not reach a significant level. Regarding the HADS, although the 25item and 23-item versions of the CS-FBD-J in the IBS group were significantly correlated with the HADSanxiety and depression scores, their intercorrelations in the control group were not significant. 
Table 4

Correlations between the CS-FBD-J (25-item and 23-item version) and other variables

\begin{tabular}{|c|c|c|c|c|c|c|c|}
\hline 25-item version & & IBS-SI & VSI & DAS-24 & $\begin{array}{l}\text { HADS } \\
\text { (Anxiety) }\end{array}$ & $\begin{array}{l}\text { HADS } \\
\text { (Depression) }\end{array}$ & $\begin{array}{l}\text { HADS } \\
\text { (Total) }\end{array}$ \\
\hline \multirow[t]{2}{*}{ Total } & $r$ & 0.617 & 0.625 & 0.249 & 0.335 & 0.219 & 0.305 \\
\hline & $p$ & $<0.001$ & $<0.001$ & $<0.001$ & $<0.001$ & 0.001 & $<0.001$ \\
\hline \multirow[t]{2}{*}{ IBS group } & $r$ & 0.569 & 0.553 & 0.213 & 0.455 & 0.361 & 0.444 \\
\hline & $p$ & $<0.001$ & $<0.001$ & 0.035 & $<0.001$ & $<0.001$ & $<0.001$ \\
\hline \multirow[t]{2}{*}{ Control group } & $r$ & 0.488 & 0.515 & 0.224 & 0.162 & 0.144 & 0.169 \\
\hline & $p$ & $<0.001$ & $<0.001$ & 0.017 & 0.084 & 0.127 & 0.073 \\
\hline \multirow[t]{2}{*}{ 23-item version } & & IBS-SI & VSI & DAS-24 & HADS & HADS & HADS \\
\hline & & & & & (Anxiety) & (Depression) & (Total) \\
\hline \multirow[t]{2}{*}{ Total } & $r$ & 0.620 & 0.632 & 0.221 & 0.336 & 0.222 & 0.307 \\
\hline & $p$ & $<0.001$ & $<0.001$ & 0.001 & $<0.001$ & 0.001 & $<0.001$ \\
\hline \multirow[t]{2}{*}{ IBS group } & $r$ & 0.574 & 0.557 & 0.191 & 0.450 & 0.357 & 0.440 \\
\hline & $p$ & $<0.001$ & $<0.001$ & 0.059 & $<0.001$ & $<0.001$ & $<0.001$ \\
\hline \multirow[t]{2}{*}{ Control group } & $r$ & 0.492 & 0.531 & 0.179 & 0.167 & 0.150 & 0.175 \\
\hline & $p$ & $<0.001$ & $<0.001$ & 0.058 & 0.075 & 0.111 & 0.062 \\
\hline \multicolumn{8}{|c|}{ IBS: irritable bowel syndrome } \\
\hline \multicolumn{8}{|c|}{ CS-FBD-J: Japanese version of Cognitive Scale for Functional Bowel Disorders } \\
\hline \multicolumn{8}{|c|}{ IBS-SI: IBS Severity Index } \\
\hline \multicolumn{8}{|c|}{ VSI: Visceral Sensitivity Index } \\
\hline \multicolumn{8}{|c|}{ DAS-24: 24-item Dysfunctional Attitudes Scale } \\
\hline \multicolumn{8}{|c|}{ HADS: Hospital Anxiety and Depression Scale } \\
\hline
\end{tabular}

\section{Criterion-related validity}

We compared the scores of the 25-item and 23-item versions of the CS-FBD-J between the IBS patient group, non-patient IBS group, and control group to confirm the criterion-related validity (Table 2). There were significant group effects in both versions of the CS-FBD-J, and multiple comparison analysis showed significant differences between all groups (i.e., IBS patient $>$ non-patient IBS > control). 


\section{Reliability}

Cronbach's a was calculated to estimate the reliability of the CS-FBD-J. The internal consistencies of the 25-item and 23-item versions (excluding items 6 and 18) in all participants, IBS group, and control group were high (25-item version: all participants: $a=0.94$, IBS group: $a=0.94$, control group: $a=0.92 ; 23$-item version: all participants: $a=0.95$, IBS group: $a=0.94$, control group: $a=0.92$ ).

The item-total correlation analysis for the 25-item and 23-item versions of the CS-FBD-J (Table 3) showed that the correlations between each item and the total score were significant (25-item version: $p \mathrm{~s}<0.001$, $r s=0.323-0.810 ; 23$-item version: $p s<0.001, r s=0.433-0.812$ ).

\section{Discussion}

While the CS-FBD-J had a one-factor structure in common with the original version, two items (items 6 and 18) the Japanese version had low factor loadings and were deleted from this version. These items of the original version were also reported to not have high factor loadings. Because the automatic thoughts referenced in these two items could not be interpreted by some people as being related to abdominal symptoms, the factor loadings of the two items were lower in this study.

We confirm that the construct validity of the CS-FBD-J is sufficient. Regardless of the presence or absence of IBS in the participants, the CS-FBD-J and IBS symptom-related scales (IBS-SI and VSI) were positively correlated. Thus, the CS-FBD-J was appropriate as a scale reflecting gastrointestinal symptomspecific cognition. In contrast, general dysfunctional cognition not specific to gastrointestinal symptoms (DAS-24) was not prominently correlated with CS-FBD-J. Although this result differs from the original version, it can be said that it clearly shows the difference between general dysfunctional cognition and IBS-specific cognition. In addition, anxiety and depression (HADS) were correlated with CS-FBD-J only in the IBS group. Since cognition of abdominal symptoms has been reported to be associated with anxiety and depression in IBS patients [3, 12], the CS-FBD-J may reflect cognition specific to gastrointestinal symptoms that affect their emotions in individuals with IBS. In both the previous study [12] and the present data, the VSI was correlated with anxiety but not with depression, whereas the CS-FBD-J was correlated not only with anxiety but also with depression. Therefore, the CS-FBD can be distinguished from the concept measured by the VSI in that it reflects cognitions that affect both anxiety and depression. The CS-FBD and the VSI can be used for different purposes or can be combined to elaborate on cognitions related to gastrointestinal symptoms.

Sufficient criterion-related validity of the CS-FBD-J was also confirmed. The CS-FBD-J scores were higher in IBS patients, non-IBS patients, and the control group, in that order. In this study, the intensity of IBS symptoms (IBS-SI) by group was also in this order. This suggests that there is a large difference in CSFBD-J scores not only in the presence or absence of IBS, but also in the high-and low-severity groups.

The high reliability of the CS-FBD-J was confirmed. The alpha coefficient was greater than 0.9 , indicating a high internal consistency. The item-total correlation was significant for all items, and exceeded 0.4 for 
the 23-item version.

The present study has several limitations. First, there were few patients with IBS among the participants in this study. In a previous study of the original version, all participants were patients with functional bowel disorder. As shown by the data of this study, the severity of IBS and non-IBS patients may differ. If we collect data from a sufficient number of patients with IBS or FBD and conduct factor analysis, the results may be different from those of this study. Second, the diagnostic criteria for IBS used in this study were not the latest Rome-IV criteria, which had not been published at the time the study was started. In the latest diagnostic criteria, the presence of abdominal pain and discomfort is essential for the diagnosis of IBS. Based on the above issues, further validation of CS-FBD-J is required by extracting individuals with IBS using the latest diagnostic criteria and collecting sufficient data from FBD patients.

\section{Conclusion}

The CS-FBD-J is a useful scale for assessing non-functional cognition specific to gastrointestinal symptoms. The present study confirmed the reliability and validity of the 23 -item version of the CS-FBS with the deletion of two items with low factor loadings. This scale is expected to contribute to a more precise assessment of patients in research on and clinical practice of functional gastrointestinal disorders.

\section{Abbreviations}

IBS: Irritable bowel syndrome

CS-FBD-J: Japanese version of Cognitive Scale for Functional Bowel Disorders

IBS-SI: IBS Severity Index

VSI: Visceral Sensitivity Index

DAS-24: 24-item Dysfunctional Attitudes Scale

HADS: Hospital Anxiety and Depression Scale

\section{Declarations}

\section{Ethics approval and consent to participate}

The present study was approved by the ethical committee of Yokohama City University (B130905050) and the National Center of Neurology and Psychiatry (A2018-046). Written informed consent was obtained from all participants.

\section{Consent for publication}


All the authors have consented to the publication of this manuscript.

\section{Availability of data and materials}

We are not able to share our data because sharing data is not approved by our ethical committees.

\section{Competing interests}

Not applicable.

\section{Funding}

This work was supported by JSPS KAKENHI (grant number 15K01685 and 19K11771).

\section{Authors' contributions}

NS organized the project, analyzed the data, and wrote the manuscript. NS, $\mathrm{HI}$, and MI designed the study. NS and FG translated the CS-FBD into Japanese. NS, YT, MF, HI, KS, TA, and MI collected the data. All the authors read, revised, and approved the final manuscript, and share the final responsibility for the decision to submit it for publication.

\section{Acknowledgments}

None.

\section{References}

1. Leibbrand R, Hiller W. Cognitive behavior therapy for functional gastrointestinal disorders: is group treatment effective? Acta Neuropsychiatr. 2003;15:242-8

2. Lackner JM, Jaccard J, Krasner SS, Katz LA, Gudleski GD, Holroyd K. Self-administered cognitive behavior therapy for moderate to severe irritable bowel syndrome: clinical efficacy, tolerability, feasibility. Clin Gastroenterol Hepatol. 2008;6:899-906

3. Sugaya N, Nomura S, Shimada H. Relationship between cognitive factors and anxiety in individuals with irritable bowel syndrome. Int J Behav Med. 2012;19:308-15

4. Toner BB, Stuckless N, Ali A, Downie F, Emmott S, Akman D. The development of a cognitive scale for functional bowel disorders. Psychosom Med. 1998;60:492-7

5. Everitt H, Landau S, Little P, Bishop FL, McCrone P, O'Reilly G et al. ACTIB trial team. Assessing Cognitive behavioural Therapy in Irritable Bowel (ACTIB): protocol for a randomised controlled trial of clinical-effectiveness and cost-effectiveness of therapist delivered cognitive behavioural therapy and web-based self-management in irritable bowel syndrome in adults. BMJ Open. 2015;5:e008622

6. Halpert A, Rybin D, Doros G. Expressive writing is a promising therapeutic modality for the management of IBS: A pilot study. Am J Gastroenterol. 2010;105:2440-8 
7. Han CJ, Dong C, Jarrett ME, Heitkemper MM. Symptom comparisons between Asian American and white American women with irritable bowel syndrome. Gastroenterol Nurs. 2018;41:223-32

8. Jarrett ME, Cain KC, Barney PG, Burr RL, Naliboff BD, Shulman R et al. Balance of autonomic nervous system predicts who benefits from a self-management intervention Program for Irritable Bowel Syndrome. J Neurogastroenterol Motil. 2016;22:102-11

9. Ljótsson B, Hesser H, Andersson E, Lackner JM, El Alaoui SE, Falk L et al. Provoking symptoms to relieve symptoms: A randomized controlled dismantling study of exposure therapy in irritable bowel syndrome. Behav Res Ther. 2014;55:27-39

10. Reme SE, StahI D, Kennedy T, Jones R, Darnley S, Chalder T. Mediators of change in cognitive behaviour therapy and mebeverine for irritable bowel syndrome. Psychol Med. 2011;41:2669-79

11. Labus JS, Bolus R, Chang L, Wiklund I, Naesdal J, Mayer EA et al. The Visceral Sensitivity Index: development and validation of a gastrointestinal symptom-specific anxiety scale. Aliment Pharmacol Ther. 2004;20(1):89-97

12. Saigo T, Tayama J, Hamaguchi T, Nakaya N, Tomiie T, Bernick PJ et al. Gastrointestinal specific anxiety in irritable bowel syndrome: validation of the Japanese version of the visceral sensitivity index for university students. Biopsychosoc Med. 2014;8(1):10

13. Kanazawa M, Nakajima S, Oshima T, Whitehead WE, Sperber AD, Palsson OS et al. Validity and reliability of the Japanese version of the Rome III diagnostic questionnaire for irritable bowel syndrome and functional dyspepsia. J Neurogastroenterol Motil. 2015;21(4):537-44

14. Whitehead WE, Validation Working Team. Development and validation of the Rome III diagnostic questionnaire. In: association with the Rome Questionnaire Committee Drossman DA, Corazziari E, Delvaux M. Rome III: The functional gastrointestinal disorders. McLean. VA: Degnon Associates, Inc.; 2006. p. 835-65

15. Drossman DA, Corazziari E, Delvaux M, Spiller RC, Talley NJ, Thompson WG et al. Rome III: the functional gastrointestinal disorders. 3rd ed.; 2006, Degnon Associates, McLean

16. Shinozaki M, Kanazawa M, Sagami Y, Endo Y, Hongo M, Drossman DA et al. Validation of the Japanese version of the Rome II modular questionnaire and irritable bowel syndrome severity index. J Gastroenterol. 2006;41(5):491-4

17. Francis CY, Morris J, Whorwell PJ. The irritable bowel severity scoring system: a simple method of monitoring irritable bowel syndrome and its progress. Aliment Pharmacol Ther. 1997;11:395-402

18. Power MJ, Katz R, McGuffin P, Duggan CF, Lam D, Beck AT. The Dysfunctional Attitude Scale (DAS). J Res Pers. 1994;28:263-76

19. Zigmond AS, Snaith RP. The hospital anxiety and depression scale. Acta Psychiatr Scand. 1983;67:361-70

20. Zigmond AS, Snaith RP, Kitamura T. Hospital anxiety and depression scale (HAD). Arch Psychiatr Diagn Clin Eval. 1993;4:371-2 


\section{Further reading}

1. Crowne DP, Marlowe D. A new scale of social desirability independent of psychopathology. J Consult Psychol. 1960;24:349-54

2. Lackner JM, Lou Coad ML, Mertz HR, Wack DS, Katz LA, Krasner SS et al. Cognitive therapy for irritable bowel syndrome is associated with reduced limbic activity, GI symptoms, and anxiety. Behav Res Ther. 2006;44:621-38

3. Reme SE, Darnley S, Kennedy T, Chalder T. The development of the irritable bowel syndromebehavioral responses questionnaire. J Psychosom Res. 2010;69:319-25

4. Tajima M, Akiyama T, Numa $H$, Kawamura $Y$, Okada $Y$, Sakai $Y$ et al. Reliability and validity of the Japanese version of the 24-item Dysfunctional Attitude Scale. Acta Neuropsychiatr. 2007;19(6):3627

5. Tkachuk GA, Graff LA, Martin GL, Bernstein CN. Randomized controlled trial of cognitive-behavioral group therapy for irritable bowel syndrome in a medical setting. J Clin Psychol Med Settings. 2003;10:57-69

\section{Supplementary Files}

This is a list of supplementary files associated with this preprint. Click to download.

- Appendix1JapaneseversionoftheCS.docx 